\title{
Integral Quadratic Separators for performance analysis
}

\author{
Dimitri Peaucelle $^{\dagger}$, Lucie Baudouin ${ }^{\dagger}$ and Frédéric Gouaisbaut ${ }^{\dagger \ddagger}$ \\ †: LAAS-CNRS; Université de Toulouse; 7 avenue du Colonel Roche, F-31077 Toulouse, France. \\ ‡: Université de Toulouse; UPS.
}

October 15,2008

\begin{abstract}
Well-posedness of feedback connected systems is considered in topological separation framework. The case when a known linear descriptor transformation is connected to an uncertain operator is considered. Well-posedness is demonstrated to hold provided an Integral Quadratic Separator satisfying both some Linear Matrix Inequalities and an Integral Quadratic Constraint. The main result is applied to three input-output performance criteria.

Keywords: Robustness, LMI, quadratic separation, performances.
\end{abstract}

\section{Introduction}

Robust control theory of linear systems has drawn major interest of both researchers and for industrial applications these past fifteen years. The reasons are that efficient LMI-based numerical tools have been produced to guarantee stability of systems even if models are known to be inexact, but also because the developed tools provide tests for performance analysis with multiple criteria. Induced system norms such as $H_{2}$ or $H_{\infty}$ have been studied, as well as other input-to-output, state-to-output, input-to-state time-domain properties (see [1,2]). Thanks to these numerous performance formulations, results extend also to the analysis of some non-linear systems, for example for the computation of attraction domains of LTI systems with saturations (see [12]).

But all these results for performance analysis have been formalized mathematically only in the Lyapunov framework, which is one of several robust control theoretical frameworks. Integral Quadratic Constraints (IQC) [7, 6, 3] and Quadratic Separator (QS) [10, 4, 5, 9, 8] frameworks, which proved to be of major interest in terms of results with reduced conservatism, do not handle at this stage the same variety of performance criteria. Performances in the IQC framework are often limited to dissipative properties of systems, which are more general than $H_{\infty}$ and passivity, but not as general as what can be done with Lyapunov theory. These limitations are due to incompatibility of frequency domain IQC descriptions with time-domain performance specifications. QS framework handles scarcely performance criteria and if it does it is more in terms of generalization of mathematical techniques [11] than as part of that general theory.

The goal of this paper is to extend QS results to what we call Integral Quadratic Separation, IQS, and show that this formulation, not only merges the gap between IQC and QS, but also provides easy reformulations of major time-domain performance criteria. Three types of input-output performances are considered. One is norm-to-norm performance in terms of $L_{2}$ norm of signals. It is known that this " $L_{2}$ gain" performance is equivalent, for LTI systems to $H_{\infty}$ performance. The second is impulse-to-norm performance which is known to be equivalent to $H_{2}$ norm in case of LTI systems. The last considered performance is impulse-to-peak which has interpretations in terms of invariant sets.

\section{Preliminaries}

\subsection{Notations}

$\mathrm{R}^{m \times p}$ and $\mathrm{C}^{m \times p}$ are the sets of $m$-by- $n$ real and complex matrices respectively. $A^{*}$ is the transpose conjugate of the matrix $A$ (and the transpose if $A$ is real). $\mathbf{1}$ and $\mathbf{0}$ are respectively the identity and the zero matrices of appropriate 
dimensions. For Hermitian matrices, $A>(\geq) B$ means that $A-B$ is positive (semi) definite. $A^{\perp}$ is a full rank matrix whose columns span the null-space of $A$. Define as well $A^{\circledast}$ such that the columns of $\left[\begin{array}{ll}A^{\perp} & A^{\circledast}\end{array}\right]$ span $C^{m}$ (assuming $A \in \mathrm{C}^{n \times m}$ ).

Let $L_{2}^{m \times p}\left[0,+\infty\left[\right.\right.$ be the set of all measurable functions $f:\left[0,+\infty\left[\rightarrow C^{m \times p}\right.\right.$ bounded with respect to the following norm

$$
\|f\|=\operatorname{Tr}\left(\int_{0}^{\infty} f^{*}(t) f(t) d t\right)^{1 / 2}<\infty .
$$

Where $\operatorname{Tr}$ is the trace operator. Associated to that norm, is the inner product

$$
<f \mid g>=\operatorname{Tr}\left(\int_{0}^{\infty} f^{*}(t) g(t) d t\right) .
$$

To alleviate notations, in the following $L_{2}^{m \times p}[0,+\infty]$ is denoted for simplicity $L_{2}$, whatever the dimensions of the functions image set. For the ease of notations define as well the truncated inner product and norm

$$
<f\left|g>_{T}=\operatorname{Tr}\left(\int_{0}^{T} f^{*}(t) g(t) d t\right),\|f\|_{T}=<f\right| f>_{T}^{1 / 2}
$$

\subsection{Well-posedness}

Let the following interconnected system

$$
\mathcal{E} z(t)=\mathcal{A}(t) w(t), w(t)=[\nabla z](t)
$$

where $w$ and $z$ are signals describing of the system; $\mathcal{E}$ is a constant matrix, possibly not square and that may not be full rank; $\mathcal{A}$ is a time-varying matrix; $\nabla$ is an operator, that may be non-causal and may be uncertain, it is defined as belonging to a closed set $\nabla \in \nabla$. In this study, $\mathcal{A}$ is assumed almost everywhere continuous, i.e. it may be discontinuous only on a finite sequence of values $\mathcal{T}=\left\{t_{k}\right\}_{k=1 \ldots \infty}$. Moreover, $\mathcal{A}$ is assumed to have bounded entries.

The interconnected system (1) is said to be well-posed if for all operators $\nabla \in \nabla$ the internal vectors characterizing the system are unique and bounded. More specifically, consider the decomposition of $z$ in the $\left[\begin{array}{ll}\mathcal{E}^{\perp} & \mathcal{E}^{\circledast}\end{array}\right]$ basis, i.e. $z=\mathcal{E}^{\perp} y_{1}+\mathcal{E}^{\circledast} y$. With these notations, the feedback connected system writes as

$$
\mathcal{E E}^{\circledast} y=\mathcal{A} w, w=\nabla \mathcal{E}^{\perp} y_{1}+\nabla \mathcal{E}^{\circledast} y .
$$

As $\nabla$ may be rank-deficient, the vector $y_{1}$ may be non-unique and unbounded, at least for some values of $\nabla$. The vector $y_{1}$ is therefore not an internal variable of the system but rather a perturbation, possibly unbounded. The definition of well-posedness of the feedback connected system is therefore based on proving that for all uncertainties $\nabla \in \nabla$ the internal variables $w$ and $y$ are unique and bounded. Note as well that $\mathcal{E} z=\mathcal{E} \mathcal{E}^{\circledast} y$ is bounded if and only if $y$ is bounded. Therefore the $y$ signal is replaced by $\mathcal{E} z$ in the following well-posedness definition.

Definition 1 The interconnected systems (1) is said to be well-posed if whatever bounded signals $(\bar{z}, \bar{w})$ perturbating the system equations

$$
\mathcal{E} z(t)=\mathcal{A}(t) w(t)+\bar{z}(t), w(t)=[\nabla z](t)+\bar{w}(t)
$$

and whatever uncertain operator $\nabla \in \nabla$, the internal signals $(\mathcal{E} z, w)$ are unique and bounded in $L_{2}$ :

$$
\exists \bar{\gamma}>0:\left\|\begin{array}{c}
\mathcal{E} z \\
w
\end{array}\right\| \leq \bar{\gamma}\left\|\begin{array}{c}
\bar{z} \\
\bar{w}
\end{array}\right\|, \quad \begin{gathered}
\quad \forall\left(\begin{array}{c}
\bar{z} \\
\bar{w}
\end{array}\right) \in L_{2}, \\
\forall \nabla \in \nabla
\end{gathered}
$$

To illustrate the definition consider the following closed-loop $E \dot{x}=A x, x=\mathcal{I}_{1} \dot{x}$ where $\mathcal{I}_{1}$ is the integration operator with zero initial conditions:

$$
\left[\mathcal{I}_{1} \dot{x}\right](t)=\int_{0}^{t} \dot{x}(\tau) d \tau=x(t)
$$


Well-posedness of the loop implies that the only solution is identically zero $(x=0$ defines the unique equilibrium point, the descriptor system is regular). Well-posedness implies the system is impulse-free: whatever bounded, maybe discontinuous disturbances, the internal signals are bounded thus cannot be impulses. Moreover, the equilibrium is stable: for bounded perturbations, the solution is unique and bounded. Well-posedness therefore implies the system is admissible (and the converse holds as well, see [8]).

Theorem 1 The interconnected system (1) is well posed if there exists a Hermitian time-varying matrix $\Theta(t)$ with bounded entries satisfying simultaneously the LMIs:

$$
\left[\begin{array}{ll}
\mathcal{A}^{*}(t) & \mathbf{1}
\end{array}\right] \Theta(t)\left[\begin{array}{c}
\mathcal{A}(t) \\
\mathbf{1}
\end{array}\right]>\mathbf{0}, \forall t \in[0,+\infty[\backslash \mathcal{T}
$$

at all instants $t$, excepting discontinuities, and the IQC:

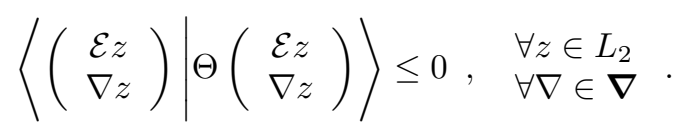

Proof: Since $\mathcal{A}$ and $\Theta$ have bounded entries, condition (4) implies that there exists a positive scalar $\epsilon>0$ such that for all $t \in[0,+\infty\lceil\backslash \mathcal{T}$

$$
\left[\mathcal{A}^{*}(t) \mathbf{1}\right](\Theta(t)-\epsilon \mathbf{1})\left[\begin{array}{c}
\mathcal{A}(t) \\
\mathbf{1}
\end{array}\right] \geq \mathbf{0}
$$

For signals satisfying the left-hand side equation of (2) it gives

$$
\begin{aligned}
& w^{*}(t)\left[\begin{array}{ll}
\mathcal{A}^{*}(t) & \mathbf{1}
\end{array}\right](\Theta(t)-\epsilon \mathbf{1})\left[\begin{array}{c}
\mathcal{A}(t) \\
\mathbf{1}
\end{array}\right] w(t) \\
& =\left(\begin{array}{c}
\mathcal{E} z(t)-\bar{z}(t) \\
w(t)
\end{array}\right)^{*}(\Theta(t)-\epsilon \mathbf{1})\left(\begin{array}{c}
\mathcal{E} z(t)-\bar{z}(t) \\
w(t)
\end{array}\right) \geq \mathbf{0}
\end{aligned}
$$

which taking the trace of the integral over time implies

$$
\left\langle\left(\begin{array}{c}
\mathcal{E} z-\bar{z} \\
w
\end{array}\right) \mid(\Theta-\epsilon \mathbf{1})\left(\begin{array}{c}
\mathcal{E} z-\bar{z} \\
w
\end{array}\right)\right\rangle_{T} \geq 0
$$

for all $T>0$. On the other hand, condition (5) implies for signals satisfying the right-hand side equation of (2) that for all $\nabla \in \nabla$ :

$$
\left\langle\left(\begin{array}{c}
\mathcal{E} z \\
w-\bar{w}
\end{array}\right) \mid \Theta\left(\begin{array}{c}
\mathcal{E} z \\
w-\bar{w}
\end{array}\right)\right\rangle \leq 0
$$

Hence, for a large enough $\bar{T}>0$ it implies

$$
\left\langle\left(\begin{array}{c}
\mathcal{E} z \\
w-\bar{w}
\end{array}\right) \mid \Theta\left(\begin{array}{c}
\mathcal{E} z \\
w-\bar{w}
\end{array}\right)\right\rangle_{T} \leq \frac{\epsilon}{2}\left\|\begin{array}{c}
\mathcal{E} z \\
w-\bar{w}
\end{array}\right\|_{T}^{2} .
$$

for all $T \geq \bar{T}$. Subtract (6) to (7) to get that the following holds

$$
\left\langle\left(\begin{array}{c}
\mathcal{E} z \\
w \\
\hline \bar{z} \\
\bar{w}
\end{array}\right) \mid\left[\begin{array}{cc}
\frac{\epsilon}{2} \mathbf{1} & M_{1} \\
M_{1}^{*} & M_{2}
\end{array}\right]\left(\begin{array}{c}
\mathcal{E} z \\
w \\
\hline \bar{z} \\
\bar{w}
\end{array}\right)\right\rangle_{T} \leq 0
$$

for all $T \geq \bar{T}$, where the $M_{1}$ and $M_{2}$ time-varying matrices are given by

$$
\begin{aligned}
& M_{1}(t)=(\Theta(t)-\epsilon \mathbf{1}) N_{1}-\left(\Theta(t)-\frac{\epsilon}{2} \mathbf{1}\right) N_{2} \\
& M_{2}(t)=N_{1}^{*}(\Theta(t)-\epsilon \mathbf{1}) N_{1}+N_{2}^{*}\left(\Theta(t)-\frac{\epsilon}{2} \mathbf{1}\right) N_{2}
\end{aligned}
$$

with matrices $N_{1}$ and $N_{2}$ of appropriate dimension and the following structure

$$
N_{1}=\left[\begin{array}{ll}
\mathbf{1} & \mathbf{0} \\
\mathbf{0} & \mathbf{0}
\end{array}\right], N_{2}=\left[\begin{array}{ll}
\mathbf{0} & \mathbf{0} \\
\mathbf{0} & \mathbf{1}
\end{array}\right] \text {. }
$$


Take any $\tilde{\epsilon}$ such that $\frac{\epsilon}{2}>\tilde{\epsilon}>0$ and take any $\tilde{\gamma}$ such that

$$
\tilde{\gamma} \mathbf{1}>\left(\frac{\epsilon}{2}-\tilde{\epsilon}\right)^{-1} M_{1}^{*}(t) M_{1}(t)-M_{2}(t)
$$

for all $t \geq 0$. Such $\tilde{\gamma}$ exists since the matrices $M_{1}$ and $M_{2}$ have bounded entries. A Schur complement argument implies that

$$
\left[\begin{array}{cc}
\tilde{\epsilon} \mathbf{1} & \mathbf{0} \\
\mathbf{0} & -\tilde{\gamma} \mathbf{1}
\end{array}\right] \leq\left[\begin{array}{cc}
\frac{\epsilon}{2} \mathbf{1} & M_{1}(t) \\
M_{1}^{*}(t) & M_{2}(t)
\end{array}\right]
$$

for all $t \geq 0$. This inequality combined to (8) gives

$$
\tilde{\epsilon}\left\|\begin{array}{c}
\mathcal{E} z \\
w
\end{array}\right\|_{T} \leq \tilde{\gamma}\left\|\begin{array}{c}
\bar{z} \\
\bar{w}
\end{array}\right\|_{T}
$$

for all $T \geq \bar{T}$ and for $T$ going to infinity proves well-posedness with $\bar{\gamma}=\tilde{\gamma} / \tilde{\epsilon}$.

Theorem 1 is applied in the following section for performance analysis of linear systems. The formulation allows to define these performances for descriptor systems $\mathcal{E} \neq 1$. Nevertheless, for the sake of clarity in this conference version of the paper, we shall only consider usual LTI systems, not of descriptor type. Formulas for satisfying constraints (5) are in that case easier to write and understand. The interested reader can have an insight on how to apply the results to descriptor systems in [8].

\section{Well-posedness and performance analysis}

\subsection{Norm-to-norm performance}

Let the linear descriptor system defined by the following equations

$$
\begin{aligned}
& \dot{x}(t)=A x(t)+B v(t) \\
& g(t)=C x(t)+D v(t)
\end{aligned}
$$

where $x \in \mathrm{R}^{n}$ is the state, $v \in \mathrm{R}^{m}$ is a disturbance input and $g \in \mathrm{R}^{p}$ is a performance channel output.

The norm-to-norm performance (or induced $L_{2}$-gain, which is equivalent to $H_{\infty}$ norm in case of LTI systems) is to prove, assuming zero initial conditions, that the norm of the output $g$ is bounded if the norm of the input $v$ is bounded. It reads mathematically as proving that the only admissible signals are such that

$$
\|g\| \leq \gamma \alpha \text { if }\|v\| \leq \alpha
$$

Equivalently, it corresponds to well-posedness with respect to the uncertain operator

$$
v=\hat{\nabla}_{n 2 n} g:\|v\|<\frac{1}{\gamma}\|g\|
$$

Notice that the operator is defined with a strict inequality. Since the proposed theory applies to uncertain operators defined in closed sets, the problem is slightly modified to prove well-posedness with respect to the operator

$$
v=\nabla_{n 2 n} g:\|v\| \leq \frac{1}{\gamma+\epsilon}\|g\|
$$

where $\epsilon$ is a positive infinitely small scalar. This slight modification amounts to proving that the actual norm-to-norm performance is strictly below the value $\gamma+\epsilon$.

The other operator involved in the defined problem is the upper defined integration operator for zero initial conditions $\mathcal{I}_{1}$. With these two operators, the norm-to-norm performance writes as the well-posedness of the linear equations

$$
\underbrace{\left(\frac{\dot{x}}{g}\right)}_{z}=\underbrace{\left[\begin{array}{ll}
A & B \\
C & D
\end{array}\right]}_{\mathcal{A}} \underbrace{\left(\frac{x}{v}\right)}_{w}
$$


feedback connected to the block diagonal operator

$$
\nabla=\left[\begin{array}{cc}
\mathcal{I}_{1} & \mathbf{0} \\
\mathbf{0} & \nabla_{n 2 n}
\end{array}\right]
$$

An integral quadratic separator with respect to the integration operator $\mathcal{I}_{1}$ can be chosen as

$$
\Theta_{\mathcal{I}_{1}}=\left[\begin{array}{c|c}
\mathbf{0} & -P \\
\hline-P & \mathbf{0}
\end{array}\right]: P>\mathbf{0} .
$$

Indeed, for any $T>0$ one has (recall that $x(0)=0$ )

$$
\begin{aligned}
\left\langle\left(\begin{array}{c}
\dot{x} \\
x
\end{array}\right) \mid \Theta_{\mathcal{I}_{1}}\left(\begin{array}{l}
\dot{x} \\
x
\end{array}\right)\right\rangle_{T} \\
=\operatorname{Tr}\left(-\int_{0}^{T} 2 x(t)^{*} P \dot{x}(t) d t\right) \\
=-\operatorname{Tr}\left(x^{*}(T) P x(T)\right) \leq 0 .
\end{aligned}
$$

An integral quadratic separator for the performance operator $\nabla_{n 2 n}$ can be chosen taking

$$
\Theta_{n 2 n}=\left[\begin{array}{c|c}
-\tau \mathbf{1} & \mathbf{0} \\
\hline \mathbf{0} & (\gamma+\epsilon)^{2} \tau \mathbf{1}
\end{array}\right]: \tau \geq 0 .
$$

Indeed, for any $T>0$ one has

$$
\left\langle\left(\begin{array}{l}
g \\
v
\end{array}\right) \mid \Theta_{n 2 n}\left(\begin{array}{c}
g \\
v
\end{array}\right)\right\rangle_{T}=-\tau\|g\|_{T}^{2}+\tau(\gamma+\epsilon)^{2}\|v\|_{T}
$$

It is thus negative when $T$ goes to infinity.

Combining these two integral quadratic separators one can apply Theorem 1 and get that the impulse-to-norm performance is guaranteed if condition (4) holds where

$$
\Theta=\left[\begin{array}{cc|cc}
\mathbf{0} & \mathbf{0} & -P & \mathbf{0} \\
\mathbf{0} & -\tau \mathbf{1} & \mathbf{0} & \mathbf{0} \\
\hline-P & \mathbf{0} & \mathbf{0} & \mathbf{0} \\
\mathbf{0} & \mathbf{0} & \mathbf{0} & \tau(\gamma+\epsilon)^{2} \mathbf{1}
\end{array}\right]
$$

with the constraints $P>\mathbf{0}$ and $\tau>0$.

Developing formula (4) gives the LMI constraint

$$
\left[\begin{array}{cc}
-A^{*} P-P A-\tau C^{*} C & -P B-\tau C^{*} D \\
-B^{*} P-\tau D^{*} C & \tau(\gamma+\epsilon)^{2} \mathbf{1}-\tau D^{*} D
\end{array}\right]>\mathbf{0}
$$

which is exactly (one can without conservatism choose $\tau=1$ ) the classical LMI formulation of the $H_{\infty}$ norm for LTI systems.

\subsection{Impulse-to-norm performance}

The impulse-to-norm performance is defined as proving, assuming zero initial conditions, that the sum of the norms of the outputs resulting from bounded impulses on the different inputs is below a level $\gamma$. It reads in mathematical terms as

$$
\|g\| \leq \gamma \quad \text { if } \quad v(t)=\alpha \delta(t) \mathbf{1}_{m} \quad: \quad|\alpha| \leq 1
$$

where $\delta(t) \in \mathrm{R}$ is the impulse Dirac signal and assuming in equation (9) that $x \in \mathrm{R}^{n \times m}$ is a matrix each column of which corresponds to the state system in response the impulse inputs and that $g \in \mathrm{R}^{p \times m}$ gathers in columns the $m$ performance outputs. 
Note that formula (9) is defined only for those $t$ such that the derivative of $x$ exists. Due to the impulsive assumption for $v$ the system rather writes as

$$
\begin{array}{ll}
x\left(0^{+}\right)=B \alpha & , g\left(0^{+}\right)=D \alpha \\
\dot{x}(t>0)=A x(t) & , g(t>0)=C x(t)
\end{array}
$$

In order to write these equations in terms of signals in $L_{2}$, define the following operator

$$
\varphi_{\theta}:\left\{\begin{array}{l}
L_{2} \longrightarrow L_{2} \\
x \longmapsto \varphi_{\theta} x
\end{array}\right.
$$

with properties

- $\varphi_{\theta}$ is linear,

- $\varphi_{\theta}$ is the square root of the shifted Dirac delta function, i.e. whatever $x, y$ in $L_{2}$ and whatever P:

$$
\begin{aligned}
& {\left[\varphi_{\theta} y\right]^{*}(t) P\left[\varphi_{\theta} x\right](t)=\delta(t-\theta) y^{*}(t) P x(t)} \\
& {\left[\varphi_{\theta_{1}} y\right]^{*}(t) P\left[\varphi_{\theta_{2}} x\right](t)=0 \text { if } \theta_{1} \neq \theta_{2}}
\end{aligned}
$$

By analogy to the Dirac $\delta$ operator, $\varphi_{\theta}$ can have many formal definitions. One of which being $\left[\varphi_{\theta} x\right](t)=\varphi(t-\theta) x(t)$ where $\varphi$ is the limit of complex valued functions defined as follows

$$
\varphi(t)=\lim _{\epsilon \rightarrow 0} \frac{\sqrt{\epsilon / \pi}}{t+j \epsilon}
$$

where $j^{2}=-1$. Indeed, one gets (see definition of the delta function in http://mathworld. wolfram. com/)

$$
\varphi^{*}(t) \varphi(t)=\lim _{\epsilon \rightarrow 0} \frac{\epsilon / \pi}{t^{2}+\epsilon^{2}}=\delta(t)
$$

The operator $\varphi_{0}$ allows to represent non zero initial conditions in $L_{2}$ and it is associated to the following definition of the integration operator $\mathcal{I}_{2}$ for non-zero initial conditions:

$$
\left[\mathcal{I}_{2}\left(\begin{array}{c}
\varphi_{0} x \\
\dot{x}
\end{array}\right)\right](t)=x(0)+\int_{0}^{t} \dot{x}(\tau) d \tau=x(t)
$$

With the help of these notations and definitions, the impulse-to-norm performance problem is described by four equations

$$
\begin{array}{ll}
\varphi_{0} x=B v & , \varphi_{0} g=D v \\
\dot{x}=A x & , g=C x
\end{array}
$$

and where the impulse-to-norm performance reads as

$$
\left\|\begin{array}{c}
\varphi_{0} g \\
g
\end{array}\right\| \leq \gamma \alpha \text { if } v=\alpha \varphi_{0} \mathbf{1}_{m}
$$

Equivalently, it corresponds to well-posedness with respect to the uncertain operator

$$
v=\nabla_{i 2 n}\left(\begin{array}{c}
\varphi_{0} g \\
g
\end{array}\right):\left\{\begin{array}{l}
v=\alpha \varphi_{0} \mathbf{1}_{m} \\
|\alpha| \leq \frac{1}{\gamma+\epsilon}\left\|\begin{array}{c}
\varphi_{0} g \\
g
\end{array}\right\|
\end{array}\right.
$$

where $\epsilon$ is a positive infinitely small scalar. As in the norm-to-norm performance case, the small $\epsilon$ is here for technical reasons and leads to proving that the actual impulse-to-norm performance is strictly below the value $\gamma+\epsilon$.

Gathering all exposed formulas, the impulse-to-norm performance corresponds to well-posedness property of the linear equations

$$
\underbrace{\left(\begin{array}{c}
\varphi_{0} x \\
\dot{x} \\
\varphi_{0} g \\
g
\end{array}\right)}_{z}=\underbrace{\left[\begin{array}{cc}
\mathbf{0} & B \\
A & \mathbf{0} \\
\mathbf{0} & D \\
C & \mathbf{0}
\end{array}\right]}_{\mathcal{A}} \underbrace{\left(\frac{x}{v}\right)}_{w}
$$


feedback connected to the following operator

$$
\nabla=\left[\begin{array}{cc}
\mathcal{I}_{2} & \mathbf{0} \\
\mathbf{0} & \nabla_{i 2 n}
\end{array}\right]
$$

An integral quadratic separator with respect to the integration operator $\mathcal{I}_{2}$ can be chosen as

$$
\Theta_{\mathcal{I}_{2}}=\left[\begin{array}{cc|c}
-P & \mathbf{0} & \mathbf{0} \\
\mathbf{0} & \mathbf{0} & -P \\
\hline \mathbf{0} & -P & \mathbf{0}
\end{array}\right]: P>\mathbf{0} \text {. }
$$

Indeed, for any $T>0$ one has

$$
\begin{aligned}
& \left\langle\left(\begin{array}{c}
\varphi_{0} x \\
\dot{x} \\
x
\end{array}\right) \mid \Theta_{\mathcal{I}_{2}}\left(\begin{array}{c}
\varphi_{0} x \\
\dot{x} \\
x
\end{array}\right)\right\rangle_{T_{T}} \\
& =\operatorname{Tr}\left(-x(0)^{*} P x(0)-\int_{0}^{T} 2 x(t)^{*} P \dot{x}(t) d t\right) \\
& =-\operatorname{Tr}\left(x^{*}(T) P x(T)\right) \leq 0 .
\end{aligned}
$$

An integral quadratic separator for the performance operator $\nabla_{i 2 n}$ can be chosen taking

$$
\Theta_{i 2 n}=\left[\begin{array}{cc|c}
-\tau \mathbf{1} & \mathbf{0} & \mathbf{0} \\
\mathbf{0} & -\tau \mathbf{1} & \mathbf{0} \\
\hline \mathbf{0} & \mathbf{0} & Q
\end{array}\right]: \operatorname{Tr}(Q) \leq \tau(\gamma+\epsilon)^{2}
$$

Indeed, for any $T>0$ one has due to (12) and the constaint on $\operatorname{Tr}(Q)$ :

$$
\begin{aligned}
& \left\langle\left(\begin{array}{c}
\varphi_{0} g \\
g \\
v
\end{array}\right) \mid \Theta_{i 2 n}\left(\begin{array}{c}
\varphi_{0} g \\
g \\
v
\end{array}\right)\right\rangle_{T} \\
& =-\tau\left\|\begin{array}{c}
\varphi_{0} g \\
g
\end{array}\right\|_{T}^{2}+\alpha^{2} \operatorname{Tr} Q \leq-\tau\left\|\begin{array}{c}
\varphi_{0} g \\
g
\end{array}\right\|_{T}^{2}+\tau\left\|\begin{array}{c}
\varphi_{0} g \\
g
\end{array}\right\|^{2}
\end{aligned}
$$

which is thus negative as $T$ goes to infinity.

Combining these two integral quadratic separators one can apply Theorem 1 and get that the impulse-to-norm performance is guaranteed if condition (4) holds where

$$
\Theta=\left[\begin{array}{cccc|cc}
-P & \mathbf{0} & \mathbf{0} & \mathbf{0} & \mathbf{0} & \mathbf{0} \\
\mathbf{0} & \mathbf{0} & \mathbf{0} & \mathbf{0} & -P & \mathbf{0} \\
\mathbf{0} & \mathbf{0} & -\tau \mathbf{1} & \mathbf{0} & \mathbf{0} & \mathbf{0} \\
\mathbf{0} & \mathbf{0} & \mathbf{0} & -\tau \mathbf{1} & \mathbf{0} & \mathbf{0} \\
\hline \mathbf{0} & -P & \mathbf{0} & \mathbf{0} & \mathbf{0} & \mathbf{0} \\
\mathbf{0} & \mathbf{0} & \mathbf{0} & \mathbf{0} & \mathbf{0} & Q
\end{array}\right]
$$

with the constraints $P>\mathbf{0}$ and $\operatorname{Tr}(Q) \leq \tau(\gamma+\epsilon)^{2}$.

Developing formula (4) gives a block diagonal matrix constraint

$$
\operatorname{diag}\left[\begin{array}{c}
-A^{*} P-P A-\tau C^{*} C \\
Q-B^{*} P B-\tau D^{*} D
\end{array}\right]>\mathbf{0}
$$

which, when $D=\mathbf{0}$, is exactly (one can without conservatism choose $\tau=1$ )

$$
\begin{array}{cc}
A^{*} P+P A+C^{*} C<\mathbf{0} & P>\mathbf{0} \\
Q>B^{*} P B & \operatorname{Tr}(Q) \leq(\gamma+\epsilon)^{2}
\end{array}
$$

The (infinitely small) scalar $\epsilon$ is here for technical reasons. It may as well be removed, in which case the equations are

$$
\begin{array}{cc}
A^{*} P+P A+C^{*} C<\mathbf{0} & P>\mathbf{0} \\
Q \geq B^{*} P B & \operatorname{Tr}(Q) \leq \gamma^{2}
\end{array}
$$

As expected the impulse-to-norm performance coincides with $H_{2}$ performance in the case of LTI systems with zero feed-through gain. 


\subsection{Impulse-to-peak performance}

The considered performance of system (9), is that the peak of some quadratic expression with respect to output $g$ is bounded for all bounded impulsive inputs $v$. The peak impulse is itself assumed bounded in a set defined by a quadratic expression:

$$
\max _{t \geq 0} g^{*}(t) Q_{g} g(t) \leq \gamma^{2} \quad \forall v=\delta \bar{v}, \bar{v} \in \mathrm{R}^{m}, \bar{v}^{*} Q_{v} \bar{v} \leq 1
$$

In these equations $Q_{g}$ and $Q_{v}$ are both positive semi-definite.

As explained previously, the impulse-to-peak definition has to be reinterpreted in terms of $L_{2}$ signals. It is done as follows. In the first step, define the unknown time instant $\theta$ when the quadratic cost $g^{*}(t) Q_{g} g(t)$ is maximum

$$
\theta=\arg \max _{t \geq 0} g^{*}(t) Q_{g} g(t)
$$

The cases when $\theta=0$ and $\theta>0$ are treated separately in the following. In the first case it corresponds to a peak immediately observed due to feed-through gain $D$. To separate the two cases the input $v$ is decomposed in two parts $v_{0}$ and $v_{\theta}$.

As a second step, define the truncation operator

$$
\mathbb{T}_{\theta}:\left\{\begin{array}{l}
L_{2} \longrightarrow L_{2} \\
x \longmapsto \mathbb{T}_{\theta} x
\end{array}\right.
$$

such that

$$
\begin{cases}{\left[\mathbb{\mathbb { V }}_{\theta} x\right](t)=x(t)} & \forall t \in[0 \theta] \\ {\left[\mathbb{\mathbb { T }}_{\theta} x\right](t)=0} & \forall t>\theta\end{cases}
$$

For truncated signals one has the following integration formulas

$$
\left[\begin{array}{l}
\left.\mathcal{I}_{3}\left(\begin{array}{c}
\varphi_{0} x \\
\mathbb{T}_{\theta} \dot{x}
\end{array}\right)\right] \\
\left.\mathcal{I}_{3}\left(\begin{array}{l}
\varphi_{0} x \\
\mathbb{T}_{\theta} \dot{x}
\end{array}\right)\right] \\
(t)=\mathbb{T}_{\theta} x(t), \forall t \in\left[\begin{array}{ll}
0 & \theta
\end{array}\right] \\
(t), \quad \forall t>\theta .
\end{array}\right.
$$

The integration operator for truncated signals with non-zero initial conditions $\mathcal{I}_{3}$ has an image which is a function of $\mathbb{T}_{\theta} x$ and $\varphi_{\theta} x$.

Finally, rewrite the system (9) in the following way

$$
\begin{array}{ll}
\varphi_{0} x=B v_{\theta} & , \quad \varphi_{0} g=D v_{0} \\
\mathbb{T}_{\theta} \dot{x}=A \mathbb{T}_{\theta} x & , \quad \varphi_{\theta} g=C \varphi_{\theta} x
\end{array}
$$

The impulse-to-peak performance then reads as, whatever $\theta>0$

$$
\begin{array}{llll}
g(\theta)^{*} Q_{g} g(\theta) \leq \gamma^{2} \alpha & \forall v_{\theta}=\varphi_{0} \bar{v}_{\theta} \quad: \quad \bar{v}_{\theta}^{*} Q_{v} \bar{v}_{\theta} \leq \alpha \\
g(0)^{*} Q_{g} g(0) \leq \gamma^{2} \alpha & \forall v_{0}=\varphi_{0} \bar{v}_{0} \quad: \quad \bar{v}_{0}^{*} Q_{v} \bar{v}_{0} \leq \alpha
\end{array}
$$

Equivalently, it corresponds to well-posedness with respect to the uncertain operator

$$
v=\nabla_{i 2 p, \theta} \varphi_{\theta} g:\left\{\begin{array}{l}
v=\varphi_{0} \bar{v} \\
\bar{v}^{*} Q_{v} \bar{v} \leq \frac{<\varphi_{\theta} g \mid Q_{g} \varphi_{\theta} g>}{(\gamma+\epsilon)^{2}}
\end{array}\right.
$$

which is repeated twice, once for $\varphi_{0} g$ and once for $\varphi_{\theta} g$. As in the previous performance examples, the infinitely small $\epsilon$ is introduced for technical reasons and leads to proving that the actual impulse-to-peak performance is strictly below the value $\gamma+\epsilon$.

Gathering all exposed formulas, the impulse-to-peak performance corresponds to well-posedness property of the following linear equations

$$
\underbrace{\left(\begin{array}{c}
\varphi_{0} x \\
\mathbb{T}_{\theta} \dot{x} \\
\varphi_{0} g \\
\varphi_{\theta} g
\end{array}\right)}_{z}=\underbrace{\left[\begin{array}{cccc}
\mathbf{0} & \mathbf{0} & \mathbf{0} & B \\
A & \mathbf{0} & \mathbf{0} & \mathbf{0} \\
\mathbf{0} & \mathbf{0} & D & \mathbf{0} \\
\mathbf{0} & C & \mathbf{0} & \mathbf{0}
\end{array}\right]}_{\mathcal{A}} \underbrace{\left(\begin{array}{c}
\mathbb{T}_{\theta} x \\
\frac{\varphi_{\theta} x}{v_{0}} \\
v_{\theta}
\end{array}\right)}_{w}
$$


feedback connected to the following operator

$$
\nabla=\operatorname{diag}\left[\begin{array}{lll}
\mathcal{I}_{3} & \nabla_{i 2 p, 0} & \nabla_{i 2 n, \theta}
\end{array}\right] .
$$

An integral quadratic separator with respect to the integration operator $\mathcal{I}_{3}$ can be chosen as

$$
\Theta_{\mathcal{I}_{3}}=\left[\begin{array}{cc|cc}
-P & \mathbf{0} & \mathbf{0} & \mathbf{0} \\
\mathbf{0} & \mathbf{0} & -P & \mathbf{0} \\
\hline \mathbf{0} & -P & \mathbf{0} & \mathbf{0} \\
\mathbf{0} & \mathbf{0} & \mathbf{0} & P
\end{array}\right] .
$$

Indeed, for any $T>\theta$ one has

$$
\begin{aligned}
& \left\langle\left(\begin{array}{c}
\varphi_{0} x \\
\mathbb{T}_{\theta} \dot{x} \\
\mathbb{T}_{\theta} x \\
\varphi_{\theta} x
\end{array}\right) \mid \Theta_{\mathcal{I}_{3}}\left(\begin{array}{c}
\varphi_{0} x \\
\mathbb{T}_{\theta} \dot{x} \\
\mathbb{T}_{\theta} x \\
\varphi_{\theta} x
\end{array}\right)\right\rangle_{T} \\
& =-x(0)^{*} P x(0)-\int_{0} 2 x(t)^{*} P \dot{x}(t) d t+x(\theta)^{*} P x(\theta)=0
\end{aligned}
$$

which is $\leq 0$.

An integral quadratic separator for the performance operator $\nabla_{i 2 p}$ can be chosen taking

$$
\Theta_{i 2 p}=\left[\begin{array}{c|c}
-\tau Q_{g} & \mathbf{0} \\
\hline \mathbf{0} & (\gamma+\epsilon)^{2} \tau Q_{v}
\end{array}\right]: \tau \geq 0 .
$$

Indeed, for any $T>\theta$ one has

$$
\begin{aligned}
& \left\langle\left(\begin{array}{c}
\varphi_{\theta} g \\
v
\end{array}\right) \mid \Theta_{i 2 p}\left(\begin{array}{c}
\varphi_{\theta} g \\
v
\end{array}\right)\right\rangle_{T} \\
& =-\tau g^{*}(\theta) Q_{g} g(\theta)+\tau \gamma^{2} \bar{v}^{*} Q_{v} \bar{v}
\end{aligned}
$$

which is negative by definition in (14).

Combining these two integral quadratic separators one can apply Theorem 1 and get that the impulse-to-peak performance is guaranteed if condition (4) holds where

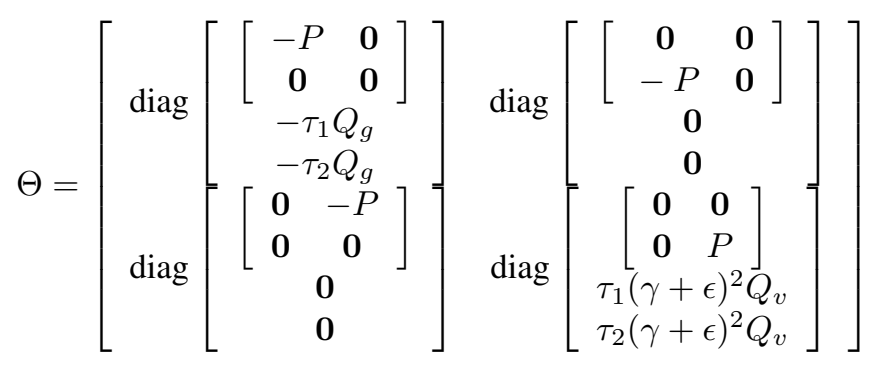

with the constraints $\tau_{1} \geq 0$ and $\tau_{2} \geq 0$.

Developing formula (4) gives a block diagonal matrix constraint

$$
\operatorname{diag}\left[\begin{array}{c}
-A^{*} P-P A \\
P-\tau_{2} C^{*} Q_{g} C \\
\tau_{1}(\gamma+\epsilon)^{2} Q_{v}-\tau_{1} D^{*} Q_{g} D \\
\tau_{2}(\gamma+\epsilon)^{2} Q_{v}-B^{*} P B
\end{array}\right]>\mathbf{0}
$$

which is exactly (one can without conservatism choose $\tau_{1}=1, \tau_{2}=1$ )

$$
\begin{array}{cc}
A^{*} P+P A<\mathbf{0} & P>C^{*} Q_{g} C \\
(\gamma+\epsilon)^{2} Q_{v}>B^{*} P B & (\gamma+\epsilon)^{2} Q_{v}>D^{*} Q_{g} D
\end{array}
$$


These should hold for some infinitely small $\epsilon$ which is the same as the set of equations with non-strict inequalities

$$
\begin{array}{cc}
A^{*} P+P A<\mathbf{0} & P \geq C^{*} Q_{g} C \\
\gamma^{2} Q_{v} \geq B^{*} P B & \gamma^{2} Q_{v} \geq D^{*} Q_{g} D
\end{array}
$$

which are exactly the LMI conditions in [1] for impulse-to-peak performance (they had considered $Q_{v}=Q_{g}=\mathbf{1}$ ).

Note that to prove a quadratic set $x^{*} Q x \leq 1$ is invariant, is a sub-case of impulse-to-peak performance where

$$
B=\mathbf{1}, C=\mathbf{1}, D=\mathbf{0}, Q_{v}=Q_{g}=Q, \gamma=1
$$

The obtained LMIs are in that case

$$
A^{*} P+P A<\mathbf{0}, P>Q,(1+\epsilon)^{2} Q>P
$$

for some infinitely small $\epsilon$. It thus gives for $\epsilon$ going to zero, that $A^{*} Q+Q A<\mathbf{0}$ as expected.

\section{Conclusion}

A new Integral Quadratic Separation result is produced and applied to provide LMI conditions for input-output performance analysis. Combined to existing robustness results developed in the Quadratic Separation framework, it paves the way for many robust performance analysis tests. Moreover, as the main result includes the case of time-varying data, extensions are expected for time-vaying systems, switched systems, fault tolerant performance evaluation, and many other topics. These extensions will be studied in future work.

\section{References}

[1] S. Boyd, L. El Ghaoui, E. Feron, and V. Balakrishnan. Linear Matrix Inequalities in System and Control Theory. SIAM Studies in Applied Mathematics, Philadelphia, 1994.

[2] L. El Ghaoui and S.-I. Niculescu, editors. Advances in Linear Matrix Inequality Methods in Control. Advances in Design and Control. SIAM, Philadelphia, 2000.

[3] M. Fu, S. Dasgupta, and Y.C. Soh. Integral quadratic constraints approach vs. multiplier approach. Automatica, 41(2):281-287, 2005.

[4] T. Iwasaki and S. Hara. Well-posedness of feedback systems: Insights into exact robustness analysis and approximate computations. IEEE Trans. on Automat. Control, 43(5):619-630, 1998.

[5] T. Iwasaki and G. Shibata. LPV system analysis via quadratic separator for uncertain implicit systems. IEEE Trans. on Automat. Control, 46(8):1195-1207, August 2001.

[6] U. Jönsson and A. Rantzer. Advances in Linear Matrix Inequality Methods in Control, chapter 6 Optimization of Integral Quadratic Constraints, pages 109-127. Advances in Design and Control. SIAM, 2000. edited by L. El Ghaoui and S.-I. Niculescu.

[7] A. Megreski and A. Rantzer. System analysis via integral quadratic constraints. IEEE Trans. on Automat. Control, 42(6):819-830, June 1997.

[8] D. Peaucelle. Quadratic separation for uncertain descriptor system analysis, strict LMI conditions. In IEEE Conference on Decision and Control, New Orleans, December 2007. Section 5 of the manuscript is erroneous, a corrected version is available at www. laas.fr/ peaucell/papers/cdc07a.pdf.

[9] D. Peaucelle, D. Arzelier, D. Henrion, and F. Gouaisbaut. Quadratic separation for feedback connection of an uncertain matrix and an implicit linear transformation. Automatica, 43:795-804, 2007. doi: 10.1016/j.automatica.2006.11.005.

[10] M.G. Safonov. Stability and Robustness of Multivariable Feedback Systems. Signal Processing, Optimization, and Control. MIT Press, 1980. 
[11] C.W. Scherer. Advances in Linear Matrix Inequality Methods in Control, chapter 10 Robust Mixed Control and Linear Parameter-Varying Control with Full Block Scallings, pages 187-207. Advances in Design and Control. SIAM, 2000. edited by L. El Ghaoui and S.-I. Niculescu.

[12] S. Tarbouriech, G. Garcia, and A.H. Glattfelder, editors. Advanced strategies in control systems with input and output constraints, volume 346 of Lecture Notes in Control and Information Sciences. Springer-Verlag, Berlin, 2007. 